\title{
Toxicity Assessments of Micro- and Nanoplastics Can Be Confounded by Preservatives in Commercial Formulations
}

\author{
Submitted to: \\ Environmental Science \& Technology Letters \\ 2018
}

Oluwadamilola Pikuda, Elvis Genbo Xu, Dimitrios Berk, \& Nathalie Tufenkji*

Department of Chemical Engineering, McGill University,

Montreal, Quebec, Canada H3A OC5

* Corresponding Author. Phone: (514)398-2999; Fax: (514)398-6678; e-mail: nathalie.tufenkji@mcgill.ca 


\section{Abstract}

Micro- and nanoplastics derived from environmental degradation of larger plastic debris can be ingested and accumulated in aquatic organisms, raising increasing global ecological concerns. Toxicology studies on aquatic organisms predominantly use commercial formulations of micro- and nano-sized polystyrene particles as model plastics. However, many of these commercially available formulations contain different preservatives, antimicrobials, or surfactants such as sodium azide, Tween 20 , and sodium dodecyl sulfate, which may introduce artifacts in toxicity assessments. In this study, we carried out acute toxicity tests on Daphnia magna, using commercial $20 \mathrm{~nm}$ and $200 \mathrm{~nm}$ polystyrene nanoparticles (PS-NPs) containing $2 \mathrm{mM}$ sodium azide as an antimicrobial preservative. The acute toxicity of non-dialyzed PS-NPs, dialyzed PS-NPs, and sodium azide alone was compared. The results reveal that the acute toxicity of the complete commercial formulation of PS-NPs was mainly associated with sodium azide and not the particles themselves. The dialyzed PS-NPs did not cause mortality but significantly disrupted the swimming behavior of D. magna. As commercial PSNPs are commonly and increasingly used in plastic toxicity assessments, these results highlight the importance of considering the impacts of the suspension matrix.

Keywords: plastic, polystyrene, Daphnia magna, risk assessment, additive, ecotoxicity 


\section{INTRODUCTION}

The ubiquitous presence of microplastics (MPs) in natural waters is a global concern. ${ }^{1}$ Moreover, the likely presence of nanoplastics (NPs) that are too small to be seen with the naked eye, difficult to detect and to quantify is also of growing scientific interest. ${ }^{2-3}$ Recent studies show that MPs are bioavailable to vertebrate and invertebrate aquatic organisms. ${ }^{4-7}$ For example, Farrel and Nelson found MPs in the stomachs, gills, and ovaries of crabs after feeding them with mussels that had been earlier fed with MPs. ${ }^{8}$ However, despite the prevalence of MPs and (likely) NPs in the aquatic environment, there is limited information available about the potential health impacts following ingestion. ${ }^{9-11}$

Daphnia magna is a non-selective feeder ${ }^{12}$ and dominant in freshwaters in nearly all continents and climate zones around the world. It has been widely used to investigate the toxicity of nanomaterials such as $\mathrm{CuO}, \mathrm{TiO}_{2}$, fullerene, $\mathrm{ZnO}$, and $\mathrm{Al}_{2} \mathrm{O}_{3}{ }^{13-19}$, as it is one of the freshwater species recommended for toxicity studies by the U.S. Environmental Protection Agency (EPA). ${ }^{20}$ Hence, it is an ideal model organism to test the toxicity of MPs and NPs upon ingestion. The effects of the uptake of MPs and NPs on D. magna have been fairly well documented. ${ }^{11,21-24}$ Polystyrene (PS) is the most common type of plastic used in D. magna toxicity assessments because of its commercial availability in the nano size range. However, suspensions of commercial polystyrene particles contain various preservatives or surfactants such as sodium azide, Tween 20 , sodium dodecyl sulfate (SDS), etc. These particles have been used in previous toxicity or exposure studies without conducting adequate control experiments to assess the effects of the additives, ${ }^{21,} 23-30$ which can be toxic to different organisms at low concentrations. ${ }^{31-34}$ Surprisingly, only few researchers washed the PS-NPs to eliminate the preservatives before their use in toxicity experiments (Table S1). ${ }^{22,35-37}$ 
Although commercial formulations of PS-NPs have been shown to cause mortality upon short-term exposure, ${ }^{24,37}$ it is unknown whether the same effect would be observed for PS-NPs in the absence of the preservatives. This study aims to investigate the effect of the presence of sodium azide (a common additive in commercial formulations of PS) on the acute toxicity of PS-NPs to D. magna neonates. The acute toxicities of commercial PS-NPs containing sodium azide, sodium azide alone, and dialyzed PS-NPs in which the sodium azide was removed were compared to identify the causative agent of the observed effects. Furthermore, a swimming assay was conducted to assess the sublethal behavioral effects of the dialyzed PS-NPs.

\section{MATERIALS AND METHODS}

\section{Polystyrene Nanoparticles (PS-NPs)}

Yellow-green fluorescently labeled carboxylated PS-NPs ( $20 \mathrm{~nm}, 505 \mathrm{~nm}$ excitation, $515 \mathrm{~nm}$ emission) and blue fluorescently labeled carboxylated PS-NPs (200 nm, $365 \mathrm{~nm}$ excitation, $415 \mathrm{~nm}$ emission) purchased from ThermoFisher were used for this study. These particles are widely used in plastic ecotoxicity studies for their ease of detection. ${ }^{11,25,27-29}$ The commercial PS-NP stock suspensions were supplied in deionized water containing $2 \mathrm{mM}$ sodium azide.

The size of the particles was confirmed using Dynamic Light Scattering (DLS) (Zetasizer NanoZS, Malvern Instruments). The DLS measurements were performed in triplicate, each containing 13 runs of 10 s. Z-average (nm) and Polydispersity Index (PDI, dimensionless) were measured to verify the size of the PS-NPs. In this study, a single batch of PS-NPs of each nominal size was used to complete all experiments. To remove sodium azide, the stock suspension was dialyzed against deionized water 
for 5 days using Spectra7 dialysis membranes, MWCO 1000. The water was exchanged every $3 \mathrm{~h}$ in the first $12 \mathrm{~h}$, and every $12 \mathrm{~h}$ for the remaining four days. The size of the particles before and after dialysis was verified using DLS. Fluorescence measurements of the suspension before and after dialysis were compared to confirm that the concentration did not significantly change as a result of dialysis (Infinite 200 PRO microplate reader, Tecan). To confirm the removal of sodium azide during dialysis, inductively coupled plasma - optical emission spectrometry (ICP-OES) (iCAP ${ }^{\mathrm{TM}} 7500$ Dual View, Thermo Scientific, UK) was used to measure the concentration of sodium ion in the non-dialyzed and dialyzed PS-NP suspensions. Sodium ion concentrations were measured instead of azide due to the methodological challenges in measuring the latter. The concentration of sodium in the final dialysis wash water was also measured. The sodium concentrations are reported in Table S2. Sodium azide powder $(\geq 99.5 \%)$ and all other chemicals for the preparation of Moderately Hard Reconstituted Water (MHRW) were purchased from Sigma-Aldrich.

\section{Acute Toxicity Test}

Daphnia magna used for this study was obtained from Environment and Climate Change Canada (Montreal) and maintained under controlled conditions in the laboratory for more than 1 year in MHRW at room temperature and a $16 \mathrm{~h}$ light, and $8 \mathrm{~h}$ dark cycle. They were fed daily with cultured green algae (Chlamydomonas reinhardtii) grown in an algae growth incubator (INFORS HT Multitron Pro) following the method described by Le Faucheur $e t$ al..$^{38}$ The MHRW used for culturing D. magna and for the toxicity assessments was prepared following the EPA guidelines for acute toxicity studies..$^{20}$ Daphnia neonates ( $<24 \mathrm{~h}$ old) were harvested and used for the acute toxicity tests. 
A standard $48 \mathrm{~h}$ acute toxicity test was conducted in compliance with the OECD guidelines (OECD, Test 202). ${ }^{39}$ Daphnia neonates were exposed to $20 \mathrm{~nm}$ and $200 \mathrm{~nm}$ PS-NPs at a series of concentrations $(0.1,1,5,10,20,50$ and $100 \mathrm{ppm})$ and observed over $48 \mathrm{~h}$. These concentrations were chosen to cover a range where no acute effects were observed until mortality was observed based on preliminary range finding tests. The concentrations are also within the range of previous toxicological studies using PS-NPs. The $100 \mathrm{ppm}$ concentration is equivalent to $1.15 \times 10^{15}$ particles $/ \mathrm{mL}$ and $1.7 \times 10^{10}$ particles $/ \mathrm{mL}$ for the $20 \mathrm{~nm}$ and $200 \mathrm{~nm}$ PS-NPs, respectively. $20 \mathrm{~mL}$ of PS-NP suspension in MHRW was prepared in $50 \mathrm{~mL}$ glass beakers. PS-NP suspensions were vortexed immediately before use. Five neonates were exposed in each beaker, and all exposures were carried out in triplicate. The animals were observed for mortality after $24 \mathrm{~h}$ and $48 \mathrm{~h}$.

Three independent exposures were carried out with different objectives. The first investigated the effect of non-dialyzed $20 \mathrm{~nm}$ and $200 \mathrm{~nm}$ PS-NPs (containing $2 \mathrm{mM}$ sodium azide) on Daphnia neonates, the second studied the effect of dialyzed $20 \mathrm{~nm}$ and $200 \mathrm{~nm}$ PS-NPs particles, and the third, the effect of sodium azide alone. The concentrations of sodium azide in the third exposure were the same as in the first exposure. Triplicated MHRW controls were carried out alongside each exposure.

\section{Swimming Assay}

After $48 \mathrm{~h}$ exposure, the swimming behavior of the animals exposed to $100 \mathrm{ppm}$ dialyzed PS-NPs as well as control conditions was observed by microscopy (10× magnification, stereomicroscope, Fisher Scientific). The swimming behavior of neonates exposed to non-dialyzed PS-NPs could not be assessed as all neonates were dead at $100 \mathrm{ppm}$. The animals were placed individually in glass bottom culture 
dishes (14 mm diameter, Matsunami Glass, VWR) filled with $1 \mathrm{~mL}$ MHRW. The light source from the microscope was placed directly above the glass bottom culture dish. The neonates were allowed to adapt to the lighting environment for 3 min before the recordings were started. For each neonate, a recording of $60 \mathrm{~s}$ was made. Ten animals were randomly selected and recorded for each treatment. The video recordings were analyzed with Kinovea software (https:/www.kinovea.org/). The swimming of the neonates over the $60 \mathrm{~s}$ period was traced and the total distance covered was recorded. Comparisons between means of swimming distance among the experimental groups were achieved by one-way analysis of variance (ANOVA) followed by the post-hoc Tukey's multiple comparison test. The level of significance was set at $\mathrm{p} \leq 0.05$.

\section{Accumulation of PS-NPs}

After the video recording, Daphnia neonates were returned into the same $50 \mathrm{~mL}$ glass beakers containing the PS-NPs for $1 \mathrm{~h}$ before being observed by fluorescence microscopy (IX71, Olympus) at $100 \times$ magnification to verify the accumulation of PS-NPs. This $1 \mathrm{~h}$ waiting period was to ensure that the animals were not stressed before imaging.

\section{RESULTS AND DISCUSSION}

\section{PS-NP Characterization}

The DLS sizes of the $20 \mathrm{~nm}$ and $200 \mathrm{~nm}$ PS-NPs were $33 \pm 8 \mathrm{~nm}$ and $263 \pm 9 \mathrm{~nm}$, respectively. This suggests that a fraction of the particle population aggregated when initially suspended in the MHRW. Hereafter, we continue to refer to the particle suspensions by their nominal sizes. DLS measurements were also conducted $48 \mathrm{~h}$ after the suspensions were prepared to verify whether the particles aggregate 
over the period of exposure. The sizes of the $20 \mathrm{~nm}$ and $200 \mathrm{~nm}$ PS-NPs were $38 \pm 16 \mathrm{~nm}$ and $278 \pm 7$ $\mathrm{nm}$, respectively after the $48 \mathrm{~h}$ period (Figure S1 in Supporting Information). This indicates that the particles remain mostly well dispersed over the $48 \mathrm{~h}$ exposure period. In addition, the total fluorescence of the PS-NP suspensions was similar before and after dialysis (Figure S2), showing that most of the particles were not lost as a result of dialysis. No sodium ion was detected in the dialyzed PS-NP suspensions (Table S2).

\section{Mortality Assessment}

Exposure to non-dialyzed PS-NPs (i.e., containing sodium azide) caused mortality starting from 10 ppm and reached 100\% at $100 \mathrm{ppm}$ for both $20 \mathrm{~nm}$ and $200 \mathrm{~nm}$ PS-NPs (Figure 1). The results showed significant mortality after $48 \mathrm{~h}$ exposure for both $20 \mathrm{~nm}$ and $200 \mathrm{~nm}$ PS-NPs with LC50 of $15.7 \mathrm{ppm}$ (equivalent to $3.0 \times 10^{14}$ particles $/ \mathrm{mL}$ ) and $30.4 \mathrm{ppm}$ (equivalent to $1.6 \times 10^{10}$ particles $/ \mathrm{mL}$ ), respectively (Fig. 1c, d). Mortality was also observed for neonates exposed to sodium azide at the same concentration as that present in the non-dialyzed PS-NPs with LC50 of 13.4 ppm (Fig. 1e). The mortality observed for sodium azide alone was very similar to that observed for the $20 \mathrm{~nm}$ PS-NPs at all concentrations and slightly different for the $200 \mathrm{~nm}$ PS-NPs between $5 \mathrm{ppm}$ and 20 ppm (Figure 1). In contrast, dialyzed PS-NPs caused no mortality at all concentrations for both $20 \mathrm{~nm}$ and 200 nm PS-NPs (Figure 1). This agrees with some earlier studies exposing aquatic organisms to dialyzed PS-NPs. ${ }^{22,36}$ For instance, Mattsson $e t a l^{22}$ observed no mortality of D. magna exposed to dialyzed PSNPs up to $24 \mathrm{~h}$. Similarly, Cole and Galloway ${ }^{36}$ did not observe any mortality when dialyzed PS-NPs were fed to Pacific oyster larvae. The observed survival of D. magna neonates upon exposure to dialyzed 
PS-NPs contradicts the results of Mattsson et al. ${ }^{37}$ and Aljaibachi and Callaghan ${ }^{24}$ who reported mortality when Daphnia was exposed to PS-NPs. In Aljaibachi and Callaghan's study ${ }^{24}$, adult Daphnia were fed with $146 \mathrm{ppm}$ MPs and mortality was observed within $72 \mathrm{~h}$. However, the particles were not dialyzed but were rather used as purchased. Mattsson $e t$ al. ${ }^{37}$ reported mortality when Daphnia was exposed to $75 \mathrm{ppm} 52 \mathrm{~nm}$ PS-NPs for $24 \mathrm{~h}$, but it is important to note that the particles used for the study were dialyzed for $24 \mathrm{~h}$ only, which is far shorter than the 5 days of dialysis used in the present study.

\section{Swimming Behavior of Daphnia Neonates}

To reveal the sublethal effects of PS-NPs, the swimming behavior of the neonates after exposure to dialyzed PS-NPs as well as control neonates was assessed. The dialyzed $20 \mathrm{~nm}$ and $200 \mathrm{~nm}$ PS-NPs showed no acute toxicity at $100 \mathrm{ppm}$ but significantly affected the swimming behavior of Daphnia when compared to the control neonates. The swimming behavior of neonates exposed to non-dialyzed PS-NPs could not be assessed as all neonates were dead at this concentration. As shown in Figure 2, the swimming activity was low for the control neonates, but significantly higher for the neonates exposed to dialyzed PS-NPs. Neonates exposed to PS-NPs swam up to $40 \mathrm{~cm}$ in one min while the neonates from the control experiments barely moved up to $10 \mathrm{~cm}$ during the same time period. For the $200 \mathrm{~nm}$ PS-NPs, the animals demonstrated more activity during the 1 min period than the $20 \mathrm{~nm}$ PS-NPs suggesting that particle size can be an important factor for the toxicity of PS-NPs as suggested

earlier by Mattsson $e t$ al. ${ }^{37}$ Daphnia exhibiting a hyperactive swimming behavior and demonstrating longer swimming distance within a given period of time are more likely to be exposed to predators, 
and thus at higher ecological risk. ${ }^{40} \mathrm{It}$ is interesting to note that the observed hyperactive swimming behavior upon exposure to PS-NPs is similar to that noted with other nanomaterials such as nano- $\mathrm{C}_{60}$ and the hydrogenated $\mathrm{C}_{60}-\mathrm{H}_{\mathrm{x}} \mathrm{C}_{70} \mathrm{H}_{\mathrm{x}}{ }^{40}$ suggesting possible shared toxicity mechanisms of these nanoparticles. Further studies are needed to investigate the molecular mechanisms of behavioral toxicity of different nanoparticles.

\section{Ingestion and Uptake into the Gastro-Intestinal Tract (GIT)}

Fluorescence images of the neonates were taken after $48 \mathrm{~h}$ exposure to the PS-NPs to ascertain whether the neonates ingested the dialyzed PS-NPs even though no mortality was observed. The images show the uptake of both non-dialyzed and dialyzed PS-NPs in the gastrointestinal tract (GIT) of the animals (Figure 3). The images of the neonates exposed to $100 \mathrm{ppm}$ of $20 \mathrm{~nm}$ and $200 \mathrm{~nm}$ dialyzed PS-NPs show significant uptake of the particles in the GIT without any visible damage. In contrast, neonates exposed to the same concentration of non-dialyzed PS-NPs after $48 \mathrm{~h}$ show that the PS-NPs were not only ingested, but there was also visible rupture of the GIT (Figure 3b and 3d). This indicates that the dialyzed PS-NPs were ingested by the neonates and present in the GIT even though no mortality was observed, and the visible rupture observed in the GIT of the neonates exposed to non-dialyzed PSNPs is likely linked to the sodium azide, which may explain the high mortality observed in the sodium azide exposure. 
In summary, this study is the first to show that the observed mortality of D. magna can be directly attributed to a preservative (in this case, sodium azide) present in a PS-NP suspension. After the removal of sodium azide by dialysis, the PS-NPs caused no mortality but significantly affected the mobility of the neonates. Interestingly, of the studies conducted using commercial stock suspensions of PS-NPs, a considerable number exhibit higher toxicity than those where the plastic particles were washed (Table S1). Even though stabilizers are very useful in the preparation and preservation of engineered nanoparticles, the presence of these stabilizers may be misleading and cause over- or underestimation of particle toxicity, ${ }^{34}$ and there is no exemption for commercial micro- and nanoplastics. As the number of toxicology studies on micro- and nanoplastics continues to increase, the results of this study call for a more careful assessment of the toxic effects of the additives in commercial particle formulations. 


\section{Acknowledgements}

The authors acknowledge the financial support of the Department of Fisheries and Oceans, Canada, the Natural Sciences and Engineering Research Council of Canada, the Canada Foundation for Innovation, and a McGill Engineering Doctoral Award to OP. The authors thank Magali Houde, Maeva Giraudo and Geneviève Farley at Environment and Climate Change Canada for providing the D. magna and advice on Daphnia rearing, and Nariman Yousefi and Jeffrey Farner for their assistance and helpful discussions.

\section{ASSOCIATED CONTENT}

\section{Supporting Information Available:}

Dynamic Light Scattering (DLS) measurements (Figure S1), fluorescence (counts per second) measurements of PS-NP suspensions (Figure S2), a summary of studies on the toxicity of PS-NPs to different organisms (Table S1), and the concentration of sodium ion in PS-NP suspensions and final dialysis wash water (Table S2). 


\section{Literature Cited}

1. Alimi, O. S.; Farner Budarz, J.; Hernandez, L. M.; Tufenkji, N., Microplastics and nanoplastics in aquatic environments: aggregation, deposition, and enhanced contaminant transport. Environmental Science \& Technology 2018, 52 (4), 1704-1724.

2. Hernandez, L. M.; Yousefi, N.; Tufenkji, N., Are there nanoplastics in your personal care products? Environmental Science \& Technology Letters 2017, 4 (7), 280-285.

3. Mintenig, S. M.; Bauerlein, P.; Koelmans, A. A.; Dekker, S. C.; van Wezel, A., Closing the gap between small and smaller: Towards a framework to analyse nano-and microplastics in aqueous environmental samples. Environmental Science: Nano 2018.

4. Tanaka, K.; Takada, H.; Yamashita, R.; Mizukawa, K.; Fukuwaka, M.-A.; Watanuki, Y., Accumulation of plastic-derived chemicals in tissues of seabirds ingesting marine plastics. Marine Pollution Bulletin 2013, 69, 219-222.

5. Moos, N. V.; Burkhardt-Holm, P.; Köhler, A., Uptake and effects of microplastics on cells and tissue of the blue mussel Mytilus edulis L. after an experimental exposure. Environmental Science \& Technology 2012, 46 11327-11335.

6. Lusher, A. L.; McHugh, M.; Thompson, R. C., Occurrence of microplastics in the gastrointestinal tract of pelagic and demersal fish from the English Channel. Marine Pollution Bulletin 2013, 6794-99.

7. Rebolledo, E. L. B.; Franeker, J. A. V.; Jansen, O. E.; Brasseur, S. M. J. M., Plastic ingestion by harbour seals (Phoca vitulina) in The Netherlands. Marine Pollution Bulletin 2012, 67 200-202.

8. Farrel, P.; Nelson, K., Trophic level transfer of microplastic: Mytilus edulis (L.) to Carcinus maenas (L.). Environmental Pollution 2013, 177 1-3.

9. Besseling, E.; Wang, B.; Lürling, M.; Koelmans, A. A., Nanoplastic Affects Growth of $S$. obliquus and Reproduction of D. magna. Environmental Science \& Technology 2014, 48 (20), 1233612343.

10. Nasser, F.; Lynch, I., Secreted protein eco-corona mediates uptake and impacts of polystyrene nanoparticles on Daphnia magna. Journal of Proteomics 2016, 137, 45-51.

11. Rosenkranz, P.; Chaudhry, Q.; Stone, V.; Fernandes, T. F., A comparison of nanoparticle and fine particle uptake by Daphnia magna. Environmental Toxicology and Chemistry 2009, 28 (10), 21422149.

12. DeMott, W. R., Feeding selectivities and relative ingestion rates of Daphnia and Bosmina. Limnology and Oceanography 1982, 27 (3), 518-527.

13. Lovern, S. B.; Klaper, R., Daphnia magna mortality when exposed to titanium dioxide and fullerene (C60) nanoparticles. Environmental Toxicology and Chemistry 2006, 25 (4), 1132-1137.

14. Zhu, X.; Zhu, L.; Chen, Y.; Tian, S., Acute toxicities of six manufactured nanomaterial suspensions to Daphnia magna. Journal of Nanoparticle Research 2009, 11 (1), 67-75.

15. Heinlaan, M.; Ivask, A.; Blinova, I.; Dubourguier, H.-C.; Kahru, A., Toxicity of nanosized and bulk $\mathrm{ZnO}, \mathrm{CuO}$ and $\mathrm{TiO}_{2}$ to bacteria Vibrio fischeri and crustaceans Daphnia magna and Thamnocephalus platyurus. Chemosphere 2008, 71 (7), 1308-1316.

16. Zhu, X.; Wang, J.; Zhang, X.; Chang, Y.; Chen, Y., Trophic transfer of $\mathrm{TiO}_{2}$ nanoparticles from Daphnia to zebrafish in a simplified freshwater food chain. Chemosphere 2010, 79 (9), 928-33. 
17. Zhu, X.; Chang, Y.; Chen, Y., Toxicity and bioaccumulation of $\mathrm{TiO}_{2}$ nanoparticle aggregates in Daphnia magna. Chemosphere 2010, 78 (3), 209-215.

18. Asghari, S.; Johari, S. A.; Lee, J. H.; Kim, Y. S.; Jeon, Y. B.; Choi, H. J.; Moon, M. C.; Yu, I. J., Toxicity of various silver nanoparticles compared to silver ions in Daphnia magna. Journal of Nanobiotechnology 2012, 10 (1), 14.

19. Warheit, D. B.; Hoke, R. A.; Finlay, C.; Donner, E. M.; Reed, K. L.; Sayes, C. M., Development of a base set of toxicity tests using ultrafine $\mathrm{TiO}_{2}$ particles as a component of nanoparticle risk management. Toxicology letters 2007, 171 (3), 99-110.

20. Weber, C. I., Methods for measuring the acute toxicity of effluents and receiving waters to freshwater and marine organisms. Environmental Monitoring Systems Laboratory, Office of Research and Development, US Environmental Protection Agency Cincinnati, Ohio 1991.

21. Cui, R.; Kim, S. W.; An, Y. J., Polystyrene nanoplastics inhibit reproduction and induce abnormal embryonic development in the freshwater crustacean Daphnia galeata. Scientific Reports 2017, 7(1), 12095.

22. Mattsson, K.; Ekvall, M. T.; Hansson, L. A.; Linse, S.; Malmendal, A.; Cedervall, T., Altered behavior, physiology, and metabolism in fish exposed to polystyrene nanoparticles. Environmental Science \& Technology 2015, 49 (1), 553-61.

23. Rist, S.; Baun, A.; Hartmann, N. B., Ingestion of micro- and nanoplastics in Daphnia magna - Quantification of body burdens and assessment of feeding rates and reproduction. Environmental Pollution 2017, 228, 398-407.

24. Aljaibachi, R.; Callaghan, A., Impact of polystyrene microplastics on Daphnia magna mortality and reproduction in relation to food availability. PeerJ 2018, 6, e4601.

25. Bergami, E.; Bocci, E.; Vannuccini, M. L.; Monopoli, M.; Salvati, A.; Dawson, K. A.; Corsi, I., Nano-sized polystyrene affects feeding, behavior and physiology of brine shrimp Artemia franciscana larvae. Ecotoxicology and Environmental Safety 2016, 123, 18-25.

26. Pinsino, A.; Bergami, E.; Della Torre, C.; Vannuccini, M. L.; Addis, P.; Secci, M.; Dawson, K. A.; Matranga, V.; Corsi, I., Amino-modified polystyrene nanoparticles affect signalling pathways of the sea urchin (Paracentrotus lividus) embryos. Nanotoxicology 2017, 11 (2), 201-209.

27. Della Torre, C.; Bergami, E.; Salvati, A.; Faleri, C.; Cirino, P.; Dawson, K. A.; Corsi, I., Accumulation and embryotoxicity of polystyrene nanoparticles at early stage of development of sea urchin embryos Paracentrotus lividus. Environmental Science of Technology 2014, 48 (20), 12302-11. 28. Bergami, E.; Pugnalini, S.; Vannuccini, M. L.; Manfra, L.; Faleri, C.; Savorelli, F.; Dawson, K. A.; Corsi, I., Long-term toxicity of surface-charged polystyrene nanoplastics to marine planktonic species Dunaliella tertiolecta and Artemia franciscana. Aquatic Toxicology 2017, 189, 159-169.

29. Bhattacharya, P.; Lin, S.; Turner, J. P.; Ke, P. C., Physical adsorption of charged plastic nanoparticles affects algal photosynthesis. The Journal of Physical Chemistry C 2010, 114 (39), 1655616561.

30. Lee, K. W.; Shim, W. J.; Kwon, O. Y.; Kang, J. H., Size-dependent effects of micro polystyrene particles in the marine copepod Tigriopus japonicus. Environmental Science \& Technology 2013, 47 (19), 11278-83.

31. Rosety, M.; Ordonez, F.; Rodríguez, M. R.; Rosety, J.; Carrasco, C.; Ribelles, A., Acute toxicity of anionic surfactants sodium dodecyl sulphate (SDS) and linear alkylbenzene sulphonate 
(LAS) on the fertilizing capability of gilthead (Sparus aurata L.) sperm. Histology and Histopathology 2001, 16 (3), 839-843.

32. Eskandani, M.; Hamishehkar, H.; Dolatabadi, J. E. N., Cyto/Genotoxicity Study of Polyoxyethylene (20) Sorbitan Monolaurate (Tween 20). DNA and Cell Biology 2013, 32 (9), 498503.

33. Chang, S.; Lamm, S. H., Human Health Effects of Sodium Azide Exposure: A Literature Review and Analysis. International Journal of Toxicology 2003, 22 (3), 175-186.

34. Nunes, B.; Carvalho, F.; Guilhermino, L., Acute toxicity of widely used pharmaceuticals in aquatic species: Gambusia holbrooki, Artemia parthenogenetica and Tetraselmis chuii. Ecotoxicology and Environmental Safety 2005, 61 (3), 413-419.

35. Cedervall, T.; Hansson, L. A.; Lard, M.; Frohm, B.; Linse, S., Food chain transport of nanoparticles affects behaviour and fat metabolism in fish. PLoS One 2012, 7 (2), e32254.

36. Cole, M.; Galloway, T. S., Ingestion of Nanoplastics and Microplastics by Pacific Oyster Larvae. Environmental Science \& Technology 2015, 49 (24), 14625-32.

37. Mattsson, K.; Johnson, E. V.; Malmendal, A.; Linse, S.; Hansson, L. A.; Cedervall, T., Brain damage and behavioural disorders in fish induced by plastic nanoparticles delivered through the food chain. Scientific Reports 2017, 7 (1), 11452.

38. Le Faucheur, S.; Behra, R.; Sigg, L., Phytochelatin induction, cadmium accumulation, and algal sensitivity to free cadmium ion in Scenedesmus vacuolatus. Environmental Toxicology and Chemistry 2005, 24 (7), 1731-1737.

39. OECD, Daphnia sp. acute immobilisation test: Test 202. OECD guidelines for the testing of chemicals 2004, 2.

40. Lovern, S. B.; Strickler, J. R.; Klaper, R., Behavioral and physiological changes in Daphnia magna when exposed to nanoparticle suspensions (titanium dioxide, nano- $\mathrm{C}_{60}$, and $\mathrm{C}_{60} \mathrm{HxC}_{70} \mathrm{Hx}$ ). Environmental Science \& Technology 2007, 41 (12), 4465-4470. 


\section{Figure Captions}

Figure 1. The mean survival percentage of Daphnia neonates at $48 \mathrm{~h}$ after exposure to (a) $20 \mathrm{~nm}$ nondialyzed PS-NPs, equivalent concentration of sodium azide, and $20 \mathrm{~nm}$ dialyzed PS-NPs; (b) $200 \mathrm{~nm}$ non-dialyzed PS-NPs, equivalent concentration of sodium azide and $200 \mathrm{~nm}$ dialyzed PS-NPs. Dose response curves for (c) $20 \mathrm{~nm}$ non-dialyzed PS-NPs, (d) $200 \mathrm{~nm}$ non-dialyzed PS-NPs, and (e) equivalent concentrations of sodium azide.

Figure 2. Representative swimming tracking of Daphnia neonates treated with dialyzed PS-NPs after $48 \mathrm{~h}$ exposure for (a) control (MHRW), (b) 100 ppm 20 nm PS-NPs, and (c) 100 ppm 200 nm PSNPs, respectively. (d) Average swimming distance of neonates after $48 \mathrm{~h}$ exposure for control, 100 ppm dialyzed $20 \mathrm{~nm}$ PS-NPs and 100 ppm dialyzed $200 \mathrm{~nm}$ PS-NPs. Asterisk indicates that the measurement is statistically significantly different ( $\mathrm{p}$-value $<0.05$, one-way analysis of variance (ANOVA) followed by the post-hoc Tukey's multiple comparison test) from the control $(n=10)$.

Figure 3. Daphnia neonates imaged by fluorescence microscopy after 48 h exposure to 100 ppm PSNPs: (a) $20 \mathrm{~nm}$ dialyzed PS-NPs, (b) $20 \mathrm{~nm}$ non-dialyzed PS-NPs, (c) $200 \mathrm{~nm}$ dialyzed PS-NPs, and (d) $200 \mathrm{~nm}$ non-dialyzed PS-NPs. 
Figures

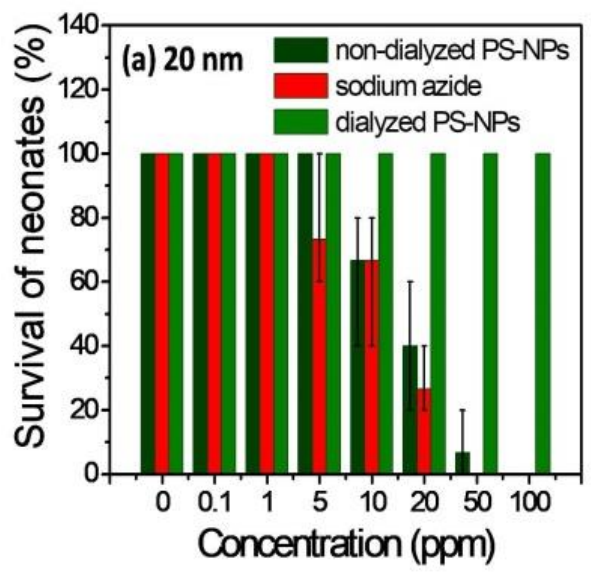

(c) $20 \mathrm{~nm}$ non-dialyzed

PS-NPS

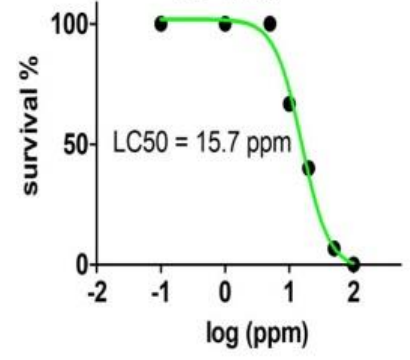

(d) $200 \mathrm{~nm}$ non-dialyzed PS-NPS

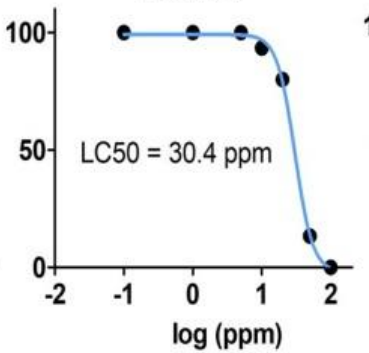

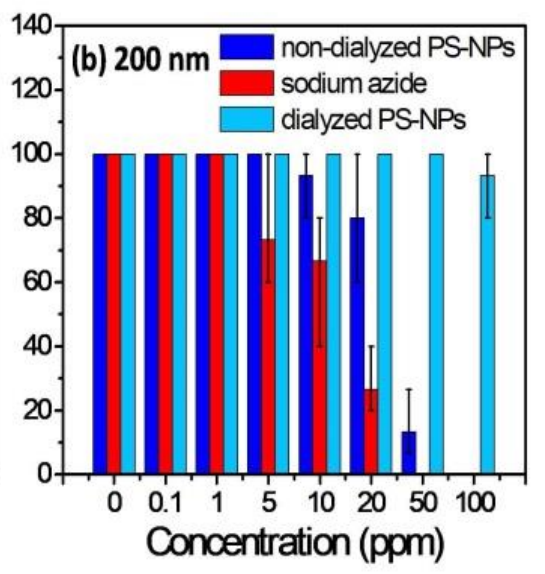

(e) sodium azide

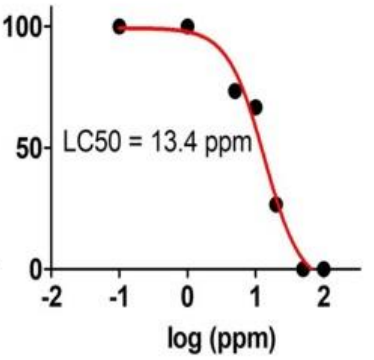

Figure 1 

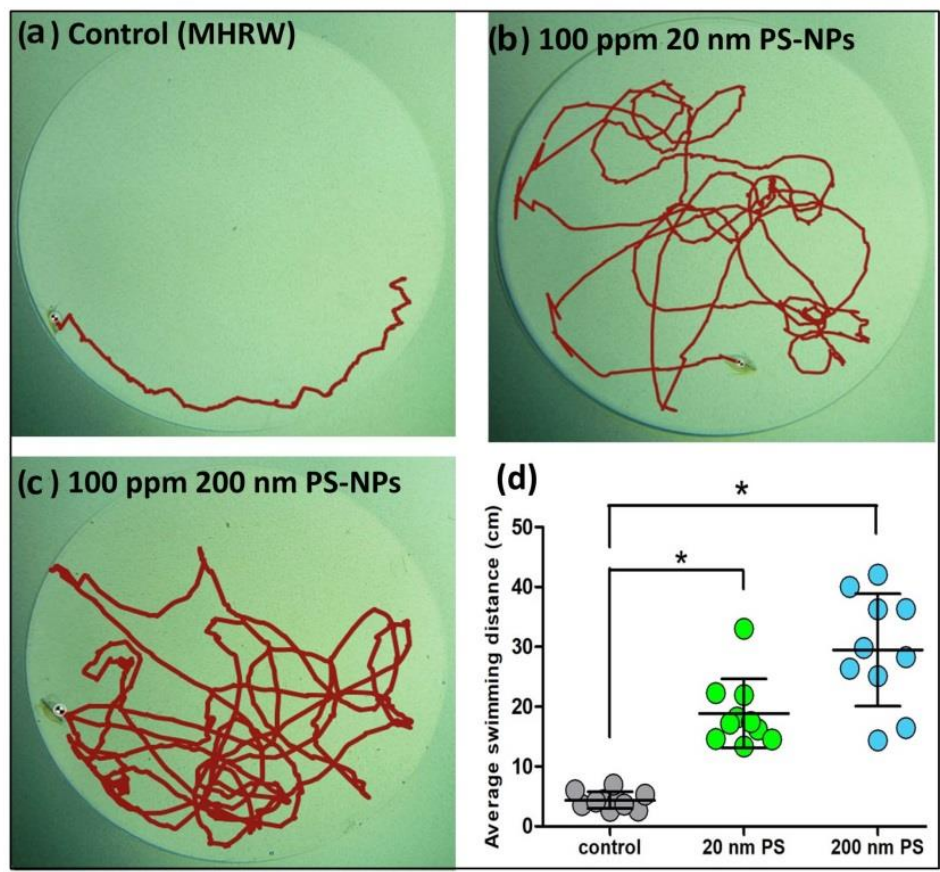

Figure 2 


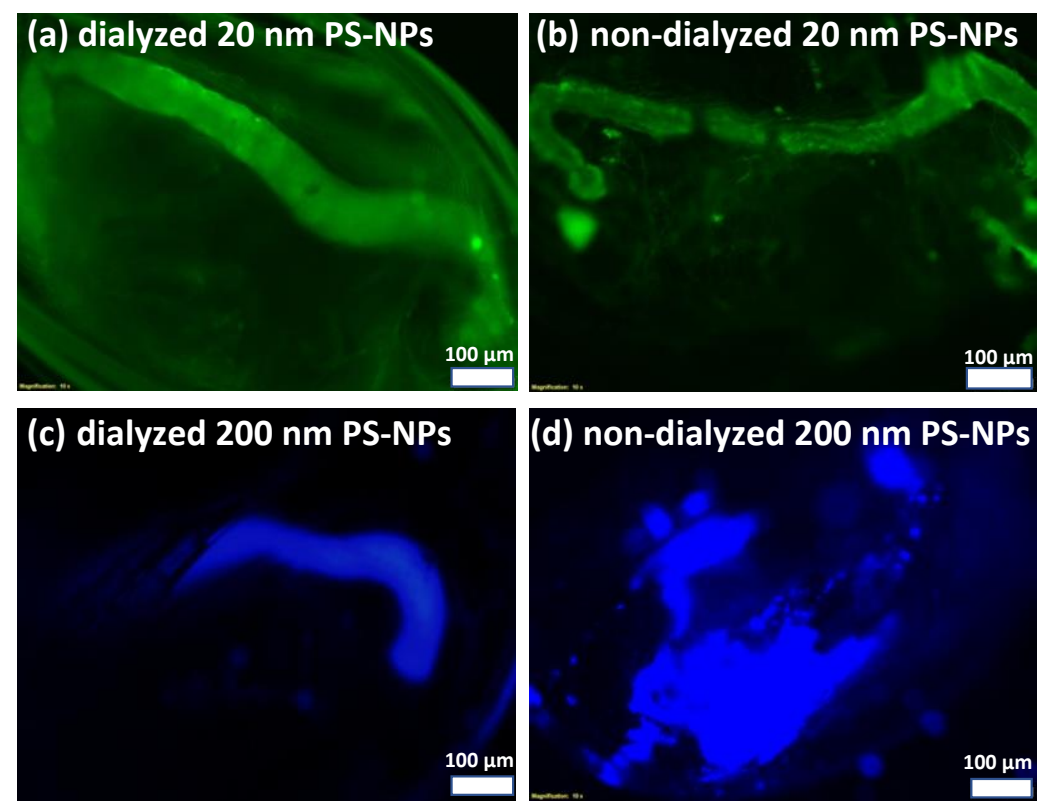

Figure 3 
TOC Graphic
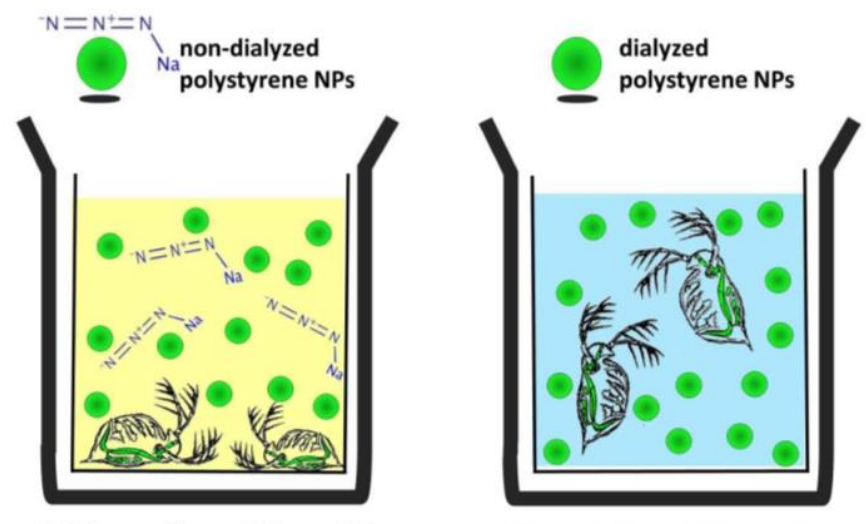

$100 \%$ mortality at $100 \mathrm{ppm}$ NPs

$0 \%$ mortality at $100 \mathrm{ppm}$ NPs 